\title{
Urinary Tract Infections in a Sabah General Hospital
}

\author{
Murtaza Mustafa ${ }^{1}$, JaistinTamin, Joseph Balingi ${ }^{2}$ \\ ${ }^{I}$ Faculty of Medicine, University Malaysia Sabah, Kota Kinabalu, Sabah, Malaysia \\ ${ }^{2}$ Microbiology Diagnostic Unit,, Queen Elizabeth Hospital, Kota Kinabalu, Sabah, Malaysia
}

\begin{abstract}
Urinary tract infections (UTI) are most common bacterial infection in women, girls and men .UTI are also prevalent in Sabah, Malaysia, and there is little information about the size of the problem amongst the local population. The aim of this study is to determine the magnitude of the problem in the hospitalized patients and community acquired infection. Over the last three years there have been 4,458 reported UTI cases. About 1,597 (35.8 \%) were Kadazans, 1,158 (26.0\%) Chinese, 319 ( $7.2 \%$ ) Bajau, 220 (5.0 \% ) Malay/Brunei, 148 (3.3\% ) Murut, 104 (2.3\% ) Bisaya, and 819 (18.4\%) were Indonesians, Filipinos and other racial ethnic groups. There was a preponderance of disease among females (female: male ratio 2.5:1) and those aged 60 years.The majority of the urinary pathogens were Escherichia coli,(38.2\%),Klebsiella,(15.0\%), and other organisms commonly isolated.The isolates were sensitive to $3^{\text {rd }}$ generation cephalosporins , amoxicillin clavulanate, and co-trimoxazole.Management of UTI requires prompt diagnosis treatment and preservation of kidney function.
\end{abstract}

Key words:. Urinary tract infection, Escherichiacoli.Klebsiella,cephalosporins,

\section{Introduction}

The infections of the urinary tract (UTIs) are among the most common bacterial infections that lead patients to seek medical care. It has been estimated that in USA more than 6 million outpatient visit and300,000 hospital stays every year due to UTIs $[1,2]$.The UTI is more common in females than among males until the age 60 when the enlargement of the prostate gland interferes with emptying of the bladder. In women between the ages of 20 and 40 who have had UTIs, as many as 50\% may become re-infected within a year.

In the UTIs bacteria can invade and cause UTI via ascending and hematogenous pathways. The ascending route is the most common route of infection in females. UTI may also occur by the hematogenous or blood borne route [3,4].In most hospitalized patients. UTI is preceded by urinary catheterization or other manipulation of the urinary tract[ 5].

Although many organisms can cause UTIs, the majority is caused by only few organisms. The organisms that infect the urinary tract are mainly strains of Escherichia coli and other Gram negative organisms[ 4].

The risk factors associated with complicated UTIs are diabetes, sickle cell anemia, kidney stones and indwelling urinary catheters[ 6] patients with UTIs are primarily young, sexually active women, who experience dysuria, frequency, and urgency but yield fewer than 105 (e.g. over 100,000) colony forming units (cfu) per ml on culture $[7,8,9,10]$.The more severe types of urinary tract infection are less common but often require hospital admission for patient management. Urinary tract infection is common among the women of age group 20-40 and in pregnancy. The frequency and impact of the infection vary in different populations. Key variables to be considered in discussing the epidemiology of urinary tract infections include gender, age, and race. Whether the urinary tract normal or abnormal, and whether infection is symptomatic or asymptomatic.

The study determines the size of the problem amongst the local population, and distribution of infection among age groups, with special emphasis on: type and frequency of urinary pathogens, antibioticsusceptibility, minimal inhibitory concentration (MIC) and multi drug resistant urinary isolates.

\section{Methods}

This was a retrospective study the data on urine culture and antimicrobial sensitivities was obtained from the record of microbiology diagnostic unit(mdu) of hospital Queen Elizabeth,KotaKinabalu,covering the period from July 2006 to June 2009. Urine samples were derived from patients in the wards and the patients attending the outpatient clinics. Urine samples were also received from the patients attending the health clinics around Kota Kinabalu.

Quantitative microbiological cultures were performed on urine samples according to standard laboratory methods. The diagnosis of clinically significant bacteriuria is performed by urinalysis and urine culture. Urinalysis is done by the dipstick test checks for the presence of leucocyte esterase, nitrites and microscopy (Johnson,1999). Quantitation of bacteriuria in cultures was accomplished by streaking a measured volume (e.g., 0.01 or $0.001 \mathrm{ml}$ ) of well mixed urine onto the surface of culture blood agar and Cystine -Lactose- 
Electrolyte Deficient (C.L.E.D) agar (Difco, Becton Dickinson, Maryland) with a calibrated dilution platinum loop. The term bacteriuria is generally used to refer to the finding of $10^{5}$ or more colony forming units(cfu) of organisms per milliliter of urine[9].

Phenotypic characterization. The 4,458 bacterial isolates were examined for their biochemical properties according to the methods described by Baer and Washington (1972) and Farmer et al(2007).

Antibiotic susceptibility test -4458 bacterial isolates were tested for antibiotic susceptibility by Bauer-Kirby disc diffusion techniques (1966),on Muller Hinton agar (Difco,Maryland).The antibiotic discs (Oxoid,Hamshire, UK) used in this assay included ampicillin (10ug) augmentin (10ug), amikacin (30ug ), ceftriaxone(30ug) cefuroxime(30ug), cefotoxime(30ug), ciprofloxacin (5ug), ceftazidime (30ug),cotrimoxazole (23.75ug), cloxacillin (1ug),erythromycin (15ug), gentamicin (10ug/120ug),imipenem (10ug),meropenem (10ug), nitrofurantoin (300ug),penicillin (10ug), tetracycyline (30ug), and vancomycin (30ug).The antibiotic assay included tests on a sensitive control organism with each batch of test strains. Staphylococcusaureus ATCC 25923 and E.coli ATCC 25922 and a standard strain of Pseudomonas aeruginosawas also used. The plates were incubated at $37 \mathrm{C}$ for $24 \mathrm{hr}$ after which inhibition zones of bacteria by the antibiotics were recorded according to the method described by Barry et al (1979).

Confirmation of methicillin resistance in Staph.aureus- Resistance to methicillin was examined by the method described by and Barber (1964).

Beta lactamase activity detection -Clinical isolates exhibiting antibiotic resistance to two or more antibiotics were examined for their resistance to penicillins or cephalosporins by the presence of beta -lactamase enzyme according to the phenotypic method, with the use of chromogenic cephalosporinase test with the cefinase disk (BD Maryland). Test was performed according to the manufacturer's instructions. The test was used for the detection of resistance to penicillin in Staph.aures, and cephalosporin resistance in Escherichia coli andKlebsiella.

Minimal inhibitory concentrations (MICs) determination -The tests were performed by using E test (AB Bio disk, Salona,Sweden) for the selected antibiotics by the method described by Bolstrom et al( 988 ).

\section{Results}

From July 2006 to June 2009, 4458 (9.6\%) urinary pathogens were isolated from 46,337 urine samples processed in the Microbiology Diagnostic Unit (MDU) (Fig.1).Pathogens were isolated from patients of all ages with peak isolation rate $1,158(26.0 \%)$ occurring in patients aged 60 years and above. Seventy percent of patients were female and thirty percent were male, the female: male ratio being 2.3: 1 . The characteristics of the studied population are shown in Table I. Urinary pathogens isolated is given in the Figure 2.Escherichia coli (38.2\%),Klebsiella(i5.0\%),Pseudomonas(9.5\%),Candidaalbicans(7.3\%),Enterobacter,Proteusmirabilis,Stphylo coccusaureus were also more commonly isolated.

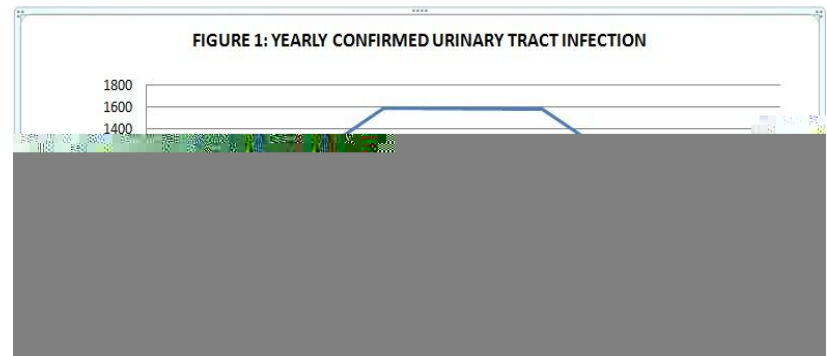

Table 1 Demographic of the study population: 4458 patients analyzed

UTI infection $4458(9.6 \%)$

Study variables*n/ $\mathrm{N} * \%$

Age

$<30 * 1366 / 4458 * 30.6$

$31-50 * 1301 / 4458 * 29.2$

$>50 * 1791 / 4458 * 40.2$

$* *$

Gender

Male*1330/4458*29.8

Female*3128/4458*70.2

**

Ethnicity

Kadazan*1597/4458*35.8

Chinese*1158/4458*26.0 
Bajau*319/4458*7.2

Malay/Brunei*220/4458*5.0

Murut*148/4458*3.3

Bisaya*104 /4458*2.3

Sungai $* 51 / 4458 * 1.1$

Rungus*42/4458*0.9

Others*819/4458*18.4

Figure 1. Distributions of main organisms isolated in MDU, HQE (June 2006 - June 2009)

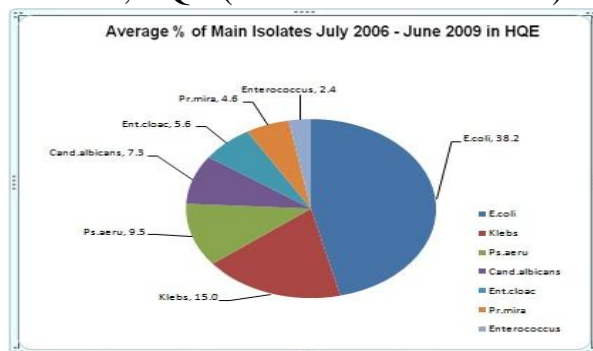

Based on July 2006-2009 hospital record.

A.

Table 2. Urinary Pathogens Isolated and Sensitivity to Selected Antibiotic

Gram Negative Organisms

\begin{tabular}{|c|c|c|c|c|c|c|c|c|c|c|c|c|c|c|}
\hline \multirow{2}{*}{ Orgoniem } & \multirow{2}{*}{$\begin{array}{l}\text { No. of } \\
\text { Patient }\end{array}$} & \multicolumn{13}{|c|}{ Antibiotic Sensitivity : } \\
\hline & & AMC & AM & $s \times T$ & FiM & GN & $\mathrm{CIP}$ & $\mathrm{CAZ}$ & MEM & IMI & exm & стх & CRO & AN \\
\hline Eschorictios coll & 1642 & 75.0 & 29.3 & 61.6 & 85.4 & 91.5 & 90.3 & \begin{tabular}{|l|l}
94.4 & \\
\end{tabular} & 94.9 & 95.1 & 83.0 & \begin{tabular}{|l|l|}
94.4 & \\
\end{tabular} & 94.1 & 96.2 \\
\hline Nabsins spp & 656 & 78.2 & 9.1 & 65.2 & \begin{tabular}{|c|}
75.2 \\
\end{tabular} & 77.7 & 68.9 & \begin{tabular}{|l|l|}
72.4 & \\
\end{tabular} & 98.5 & 100.0 & 80.1 & 72.3 & 72.7 & 77.5 \\
\hline 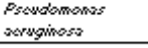 & 426 & 97.3 & 100.0 & 100.0 & 100.0 & 92.9 & 85.7 & 89.7 & \begin{tabular}{|l|l|}
88.6 \\
\end{tabular} & 88.9 & 100.0 & 100.0 & 99.1 & 89.5 \\
\hline Exerabureser & 284 & 26.1 & 14.2 & 69.6 & 49.0 & 13.2 & 51.8 & 51.8 & 13.3 & 11.9 & 77.7 & 86.9 & 90.0 & 100.0 \\
\hline Oxrobucter & 242 & 38.5 & 16.7 & 72.8 & 91.3 & 45.2 & 73.8 & 6.9 & \begin{tabular}{|l|l|} 
\\
\end{tabular} & 81.0 & 50.2 & \begin{tabular}{|l|l}
57.2 \\
\end{tabular} & 38.0 & 74.0 \\
\hline Protess minobits & 211 & 100.0 & 87.2 & 86.8 & 58.5 & 67.7 & 97.3 & 100.0 & 100.0 & 100.0 & 87.2 & \begin{tabular}{|l|l|}
96.4 \\
\end{tabular} & 96.4 & 100.0 \\
\hline 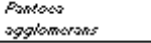 & 124 & 46.4 & 26.8 & 57.6 & 69.8 & 69.8 & 89.8 & 00.0 & 100.0 & 100.0 & 55.4 & 47.8 & 60.9 & 87.5 \\
\hline 4cimeabusterspop & 39 & 98.4 & 100.0 & 100.0 & 100.0 & 100.0 & \begin{tabular}{|l|}
98.4 \\
\end{tabular} & \begin{tabular}{|l|l}
98.4 \\
\end{tabular} & \begin{tabular}{|l|l}
98.4 \\
\end{tabular} & 98.4 & 100.0 & 89.1 & 89.1 & 99.3 \\
\hline 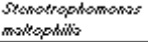 & 17 & 4.2 & 4.2 & 95.8 & 4.2 & 100.0 & 100.0 & 100.0 & 4.2 & 4.2 & \begin{tabular}{|l|}
16.7 \\
\end{tabular} & \begin{tabular}{|l|}
4.2 \\
\end{tabular} & 4.2 & 100.0 \\
\hline
\end{tabular}

B. Gram Positive Organisms

\begin{tabular}{|c|c|c|c|c|c|c|c|c|c|c|c|c|}
\hline \multirow{2}{*}{ Orgonism } & \multirow{2}{*}{$\begin{array}{l}\text { No. of } \\
\text { Patient }\end{array}$} & \multicolumn{11}{|c|}{ Antibiotic \$ensitivity \% } \\
\hline & & AMC & $\mathrm{AM}$ & $\$ X T$ & FiM & GN & $0 x$ & VA & $\mathrm{ClP}$ & P & E & $\mathrm{TE}$ \\
\hline ENeOCO & 194 & 83.5 & 29.4 & 35.9 & 45.1 & 36.9 & 95.9 & 100.0 & 36.7 & $\cdot$ & . & $\cdot$ \\
\hline Staplylococous wow & 123 & 100.0 & 7.1 & 69.0 & 96.4 & 75.1 & 75.9 & 100.0 & $\cdot$ & 100.0 & 78.8 & $\cdot$ \\
\hline Stroptocosess & 90 & 100.0 & 100.0 & 56.9 & 100.0 & 100.0 & 66.7 & 100.0 & $\cdot$ & 66.7 & 62.7 & 81.3 \\
\hline Staphyloce & 6 & & 50.0 & 100.0 & 100.0 & 100.0 & 50.0 & 100.0 & $\cdot$ & 100.0 & 66.7 & 66.7 \\
\hline
\end{tabular}

AMC,amoxicillinclavulanicacid,AM,ampicillin,SXT,cotrimoxazole,F/M,nitrofurantoin,GN,gentamicin,F/M,nitr ofurantoin,GN,gentamicin,CIP,ciprofloxacineCAZ,ceftazidime,MEM,meropenem,IMI,imipenem,CXM,cefuroxi me,CTX,cefotaxime,CRO,cefriaxone,AN,amikacin,OX,oxacillin,VA,vancomycin,P,penicillin,E,erythromycin,T E,tetracycline.

\section{Antibiotic Susceptibility Assay}

Antibiotic susceptibility patterns of 4,458 urinary isolates against 18 antibiotics is given in the Table 2(a) and(b).Among the antimicrobials that may be used for treatment of UTI in order of efficacy is third generation cephalosporin,cotrimoxazole, amoxicillin with potassium calvulanate.For Pseudomonas thirdgeneration cephalosporin is the drugs of choice.For Gram positive isolates vancomycin, amoxicillin with potassium clavulanate may be used. Resistance to vancomycin was not observed. Extended spectrum beta lactamase ESBLs was produced by 79,Escherichia coli 43 (54.4\%), Klebsiella36 (45.6\%) of 123 
Staphylococcus aureus 30 (24.4\%) were MRSA and there were 29 isolates exhibited multi drug resistance.The MIC90 of ceftriaxone against Eschericia.coli was 0.047-0.064 ugm $/ \mathrm{ml}$, of ciprofloxacin,0.006-0.047 ugm $/ \mathrm{ml}$,of gentamicin, $0.25-24 \mathrm{ugm} / \mathrm{ml}$ and of co-trimoxazole, $0.0-0.5 \mathrm{ugm} / \mathrm{ml}$.The MIC90 of ceftriaxone against Klebsiella was $0.064-24 \mathrm{ugm} / \mathrm{ml}$, of ciprofloxaxine, $0.023-128 \mathrm{ugm} / \mathrm{ml}$, of gentamicin, $0.19-24 \mathrm{ugm} / \mathrm{ml}$, and co-trimoxazole 0.032-6ugm $/ \mathrm{ml}$.Cephalosporin, and co-trimoxazole are active compared to gentamicin and ciprofloxaxine. (not given in the table).

\section{Discussion}

Urinary tract infection (UTI) is the most common bacterial infection in adults in infants and children. A UTI is defined as the detection of significant bacteria in the urine (quantitative method) with associated specific or non specificsymptoms..Australian, European and US studies vary between 2 and $13 \%$ [18] Mayo Clinic reported UTI prevalence of 2.3\%[ 19].Bristol Maternity hospital reported a UTI of 3.0\% [20].Little et al in 1966 reported UTI $5.3 \%$.Another study at Aberdeen Maternity hospital, United Kingdom 24,out of 361 antenatal cases women with previous history UTI,only $29(8.0 \%)$ developed UTI during current pregnancy[22]Retrospective studies have estimated that $1-5 \%$ infants and children will develop symptomatic UTI at least once in their life time[ $23,24,25$ ]

As observed in our study $9.6 \%$ of the subjects had UTI, which is consistent with other studies and however there is difference between our study and studies by other researchers. Other studies are mainly focused on UTI among pregnant women. In our study, UTI was observed in all age groups including the pregnant women but the peak incidence was seen in the elderly age group $(26 \%)$.

Enterobacteria mainly Escherichia coli is the most common cause of urinary tract infection and account for approximately 80-90 \% UTIs in young women, P fimbriae, organelles on strains of E. coli, are particularly virulent in that they may attach or adhere on specific receptors of uroepithelial cells and interfere with washout of bacteria. Other organisms causing UTIs include Klebsiella, Enterobacter, Staphylococcus, Proteus, Pseudomonas aeruginosa, Staphylococcus,Staphylococcussaprophyticus (usually community acquired in young sexually active women). [26,27,28]. We observed Escherichia coli isolates (38.2\%), Klebsiella(15\%), Pseudomonas aeruginosa (9.5\%), Candida albicans (7.3\%), Enterobacter (5.6\%), with other Enterobacteria in decreasing order. Staphylococcus aureus (2.5\%), and it is important to note that $24.4 \%$ of this Staphylococcus aureus were methicillin-resistant(MRSA).

In hospitalized patient the risk of bacteriuria is about $5 \%$ for each day a patient is catheterized. Up to 20 $\%$ of catheterized patients will develop bacteriuria and up to 6\% develop UTI. Fungal infections of the lower urinary tract occurs in immunocompromised patients, diabetics, and catheterized patients, and on long term antibiotics[29] Common urinary fungal pathogen is Candida albicans. In our study Candida albicans isolates account for approximately 7.3\%. However, another study done at Makati Medical Centre, Philippine, reported $3.8 \%$ Candida albicansisolates [30].

Treatment of UTI should begin only after the proper collection of a urine specimen for culture, and is based on the age of patient at diagnosis, the presenting signs and symptoms, evidence of underlying renal pathology, and presence or absence of immunologic problems[31].Choice of therapy is based either on culture and susceptibility test results or known common pathogens in the condition and their resistance patterns. Antibiotic susceptibility tests at mdu are influenced by the hospital antimicrobial policies and recommendations by the hospital drug committee. Among the antimicrobials that may be used are sulfamethoxazole-trimethoprim, amoxicillin with or without potassium clavulanate, cephalosprins, aminoglycosides, quinolones and others

This study has its limitations: Second highest UTI occurring is in the 21-30 age group, but there is lack of information on the number of pregnant and non-pregnant women?. UTI are common among the women of age group 20-40 and in pregnancy. Peak occurrence of UTI in the age group 60 years and above, no information, on patients predisposed to UTI have Diabetes, hypertension, prostatitis, immunosuppressed, prolonged hospitalization, catheterized, and nosocomial infection?. In the United States there is a $3 \%$ increase in the death rate per patient between 50-70 years was associated with the occurrence of a UTI [32]. Candida albicans isolates (7.3\%), lack of information if the Candida infection is due to other predisposing factors?. Information also lacking about boys (4\% UTI in infants and children) uncircumcised or circumcised?. Uncircumcised boys with prepuce have tendency to harbor organism in the foreskin likely due to the warm moist mucosal environment [33,34].

It is recommended that treatment of UTI include the proper collection of uncontaminated urine, identifying the infecting organisms, choosing the appropriate antimicrobials to eradicate infection and minimizing toxicity, and preventing reinfection. Prompt management of UTIs in infants, children and elderly can result in reduced morbidity and improved outcome. Further research is needed to address some of the question marks raised in this study.

\section{Conclusion}

The study from July 2006 to June 2009 reveals the incidence of UTI in 4458 patients with peak occurring in patient aged 60 years and above. Infection was more common in females and in ethnic Kadazan's. 
Escherichia coli and Klebsiella were predominant urinary pathogens. Majority of the strains isolated were susceptible to third generation cephalosporins, cotrimoxazaole.ESBLs strains and multi drug resistant strains were also detected.

\section{Acknowledgements}

The authors wish to thank Vice Chancellor University Malaysia, Sabah, Director General Ministry of Health Malaysia, Director, Health Services Sabah and Dean School of Medicine, for permission to publish this paper. We also express our appreciation to mdustafffortheir technical support. Financial assistance of UMS is gratefully acknowledged. .Ministry of Health Malaysia,,NMRR (IDN0:09-447-4152).

\section{References}

[1] Palac, DM. Urinary tract infections in women: a physician's perspective, Lab Med 17:25, 1986

[2] Wong FS: Guideline to prevention of catheter-associated urinary tract infections, Am J Infect Control 11:28,1983.

[3] Kunin CM: Urinary tract infections in females, Clin Infect Dis18:1,1994.

[4] Stamm WE et al:Urinary tract infections: from pathogens to treatment, J Infect Dis159:40,1989.

[5] Falkine FR. The insertion and management of indwelling urethral catheters: minimizing the risk of infection, $J$ Hosp Infect 25:79,1993

[6] Betty A.Forbes, Daniel F. Sahm, Alice S. Weissfeld. Bailey \& Scott's Diagnostic Microbiology, 11,ed.1998.

[7] Hamilton-Miller JMT. The urethral syndrome and its management, J Antimicrob Chemother33 (suppl A):63,1994

[8] Kunin CM, White LV, and Hua TH. A reassessment of the importance of "low count" bacteria in young women with acute urinary symptoms, Ann Intern Med119:454,1993

[9] StammWE:.Criteria for the diagnosis of urinary tract infection and for the assessment of therapeutic effectiveness, Infection20(suppl 3):S151,1992.

[10] Stamm WE. Protocol for diagnosis of urinary tract infection: reconsidering the criterion for significant bacteriuria, Urology XXX11 (suppl):6, 1988

[11] Johnson C.E.Newadvances in childhood urinary tract infection. Pediatrics in Review,20(100):333-343,19.

12] Baer, H. Washington, L. Numerical diagnostic key for the identification of Enterobacteriaceae.App.Microbiol23,108,1972

[13] Farmer JJ 111,Boatwright KK, Janda JM .Enterobacteriaceae: Introduction and Identification. In: Mannual of Clinical Microbiology,9th ed.Murray PR et al(editors).ASM press, 2007.

[14] Bauer, A. W., Kirby, W.M.M. Sherris,J.C. Truck, M. Antibiotic susceptibility testing by a standard single disc method. Am J Clin Path45:493-496,1966.

[15] Barry, A.L.,M.B. Coyle, C. Thornsberry, E.H. Gerlach,andR.W.Hawkinson.Methods of measuring zones of inhibition with BauerKirby disk susceptibility test. J Clinic Microbiol10:885-889,1979.

[16] Barber, M.(1964). Naturally occurring methicillin-resistant Staphylococci.J Gen Microb 1964;35:183.

[17] Bolstrom, A, Arvidson S, Ericson,M, Karlsson, A. A novel technique for direct quantification of antimicrobial susceptibility of microorganisms (Abst1209), Los Angeles:ICAAC,1988.

[18] Mac Lean AB.Urinary tract infection and pregnancy.In:CattelWR,editor.Infections of the kidney and Urinary Tract. .Oxford:Oxford University Pres.1966.

[19] George, D etal.A study of various tests to detect asymptomatic UTI in an Obstetric population.JAMA.1993; $16: 1971-1974$.

[20] Nunns, D, Smith ARB, Hosker G. Reagent strip testing urine for significant bacteriuria in a urodynamic UTI Clinic.BritJ Urol 1995;76:87-89.

[21] Little PJ, et al. The incidence of UTI in 5000 of pregnant women.Lancet.1966;2: 925-8

[22] Chng PK. Antenatal prediction of UTI in pregnancy. Brit J ObstetGnynecol .1982;89:8-11

[23] Hoberman, A, Chao HP, Keller DM, Hickey R, Davis HW, Ellis D. Prevalence of urinary tract infection in febrile infants. J Pediatr $1993 ; 123: 17-23$.

[24] Shaw, KN, Gorelick M, McGowan KL et al. Prevalence of UTI in febrile children in the emergency department. Pediatr 1998; 102: $1-5$.

[25] Elder, JS. Urinary tract infection, .In: Behrman RE, KliegmanRM,Jenson HB, editors. Nelson Text Book of Pediatr.16ed.Philadelphia : WB Saunders . 2000 p 1621-1625

[26] Jautin,ME,Siitonen,A.,AlaHouhala,M.,Ashron,P.,Foher,A.,Koskimies,0.,et al.Predictive factors associated with significant urinary abnormalities in infants with pyelonephritis.J Pediatric Infect Dis.2001;20(10;597-601.

[27] McCkracken,G.H. Options in antimicrobial managements of urinary tract infections in infants and children. Journal of Pediatric InfectDis.1989;8(8),552-555

[28] Hellerstein,S. Urinary tract infections in children: Pathophysiology, risk factors and managements. Infections in Medicine,2002 ;19(12),554-560.

[29] Shapiro, E.D. Infections of urinary tract. The J Pediatric Infec Dis.1992;11(20,165-168

[30] Rebecca Littaua, Thelma Tupasi. Nosocomial Infections at the Makati Medical Centre: Prospective Study and Analysis of Risks factors. Phil J Infec Dis. 1986;15(1):1-6.

[31] Nelson, D.S., Gurr,MB., Schunk, J.E. Management of febrile children with urinary tract infections. American Journal of Emergency Medicine1998;16 (7),643-647

[32] PatchenDellinger,E. Critical Care. Nosocomial Infection: ACS Surgery online.2002; WebMD Inc. Posted 06.07.2006.

[33] Herzog LW. Urinary tract infections and circumcision: a case control study. Am J Dis Child 1989:143(3):648-50.

[34] WiswellTE,Roscelli JD. Corroborative evidence for the decreased incidence of urinary tract infections in circumcised male infants. Pediatric 1986;78(1):96-9. 